\title{
Recorded Magnetization Distributions in Thin Film Disk Media
}

\author{
B. K. Middleton, M. Aziz, M. Wdowin, and J. J. Miles
}

\begin{abstract}
The magnetization distributions in recorded transitions in thin film disk media are shown to consist of simple longitudinal magnetization transitions along with flux closure patterns. Together they give rise to the familiar sawtooth patterns but also indicate why arctangent and other approximations used in record theory have proved so successful even when sawteeth are known to be present.
\end{abstract}

Index Terms-Disk media, magnetic recording, magnetization distributions.

\section{INTRODUCTION}

I T IS NOW well established that recorded magnetization transitions in thin film disk media take on a form which is often referred to as zig-zag or sawtooth. There is an abundance of experimental evidence describing the nature of these transitions [1]-[4], and it is regularly assumed that the sawtooth nature arises through a process of energy reduction. There is also evidence that the energy reduction process leads to the formation of flux closure patterns or vortices within transitions [5]. The presence of such transitions has also been predicted by micromagnetic modeling [6], [7], and these predictions have shown a great similarity to the experimental observations [1]-[3].

Despite a growing background of knowledge of the existence of sawtooth structures, record theories based on transitions with simple one-dimensional forms, such as arctangent [4], [8], tanh, or error function [8], have over the years persistently been successful in predicting many experimental observations. Concerns about the limitations of such theories has led to a number of theoretical treatments of recording sawtooth patterns [9]-[11], and rather surprisingly, these have shown results which are broadly similar to those obtained using arctangent theory when describing written transitions widths [9]-[11]. Even as transition widths have been reduced and more complicated micromagnetic structures have been apparent within transitions [5], there has been a continued use of arctangent theories which have remained at least superficially successful in predicting recording properties.

Noise in thin film media has long been said to be associated with distributions of sawtooth amplitudes and locations. However, the successful theories of noise are still based on the assumptions of arctangent transitions [12]-[14], and these

Manuscript received February 5, 1998; revised April 17, 1998. This work was supported in part by a grant and Research Studentship from the Engineering and Physical Sciences Research Council.

The authors are with the Division of Electrical Engineering, University of Manchester, Manchester M13 9PL U.K. (e-mail: b.middleton@man.ac.uk).

Publisher Item Identifier S 0018-9464(98)05042-0. have been shown to have good agreement with experimental observations of both noise spectra and total noise powers, at least until the onset of supra-linear noise at high densities.

Therefore, it seems, overall, that while the sawtooth nature of transitions is very evident, the use of arctangent theory has persisted and that even in circumstances where the transitions do not at least superficially appear to be of arctangent nature, the arctangent theory still enjoys some success. It would seem proper to ask why this is the case, and the aim of this paper is to discuss the nature of transitions and give some background to the success of analytical record theory.

\section{Micromagnetic PRedictions}

Magnetization distributions within recorded transitions have been produced by micromagnetic modeling of a granular structured thin film [15]. Results described here are for a film of thickness $30 \mathrm{~nm}$, with cylindrical grains of circular crosssection disposed on a hexagonal lattice and with diameter $25 \mathrm{~nm}$, with no exchange coupling between grains, with anisotropy axes distributed randomly in three dimensions, with magnetization $1.5 \times 10^{6} \mathrm{~A} / \mathrm{m}$, and with a packing fraction 0.777. Fig. 1 shows the distribution at a recorded transition and its breakdown into various components. Fig. 1(a) represents the variation of the magnetization component parallel to the recorded track across a sequence of transitions and here the familiar sawtooth nature is strongly evident. This has been broken down into the curl-free and divergence-free components [15] of magnetization, in the manner described by Beardsley [16], via the compution of Lorentz differential phase contrast (DPC) and field [in this case magnetic force microscope (MFM)] imagings. Fig. 1(b) shows the curl-free components of magnetization and Fig. 1(c) the divergence-free components. It is perhaps surprising that the magnetization of the transition breaks down so distinctly into two components where the divergence-free component shown in Fig. 1(c) contains all, or virtually all, of the sawtooth structure leaving the curl-free component with relatively little "decoration." This leads to the unexpected conclusion that the superstructure of the transition, that part which gives rise to the sawteeth, is divergence-free and that what is left which is not divergencefree is a very "orderly" longitudinal magnetization transition.

It is clear that the sawteeth are only a manifestation of energy reduction within the transitions through the production of flux-closure patterns and produce no fields. The remaining component of the magnetization, which could approximately be represented by an arctangent, tanh, error, or other simple function, if so wished, is the field-producing part of the 


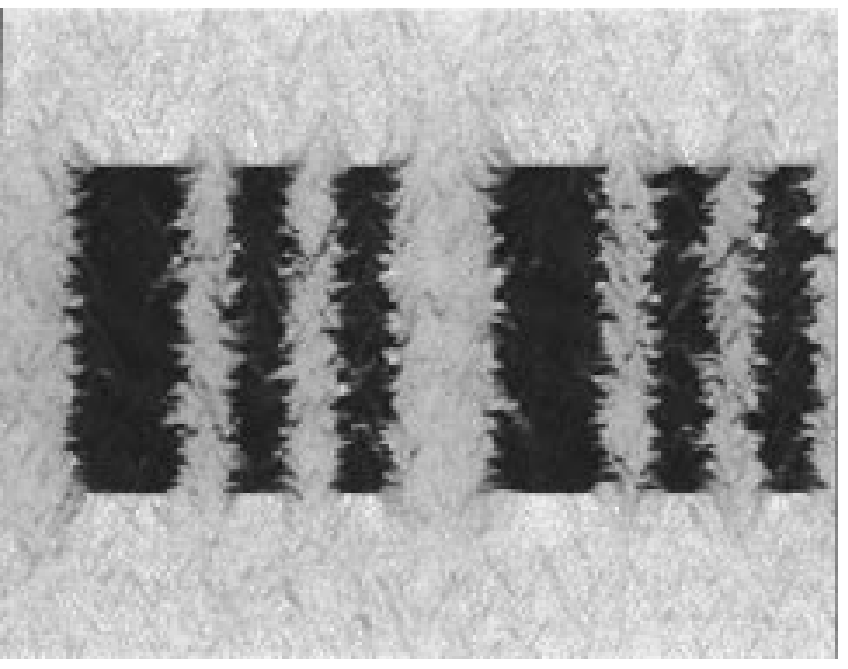

(a)

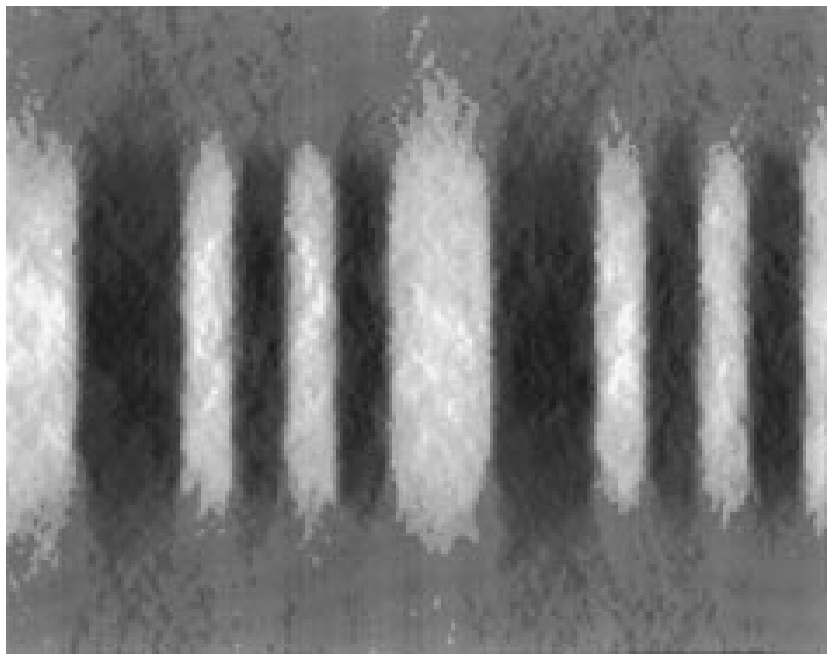

(b)

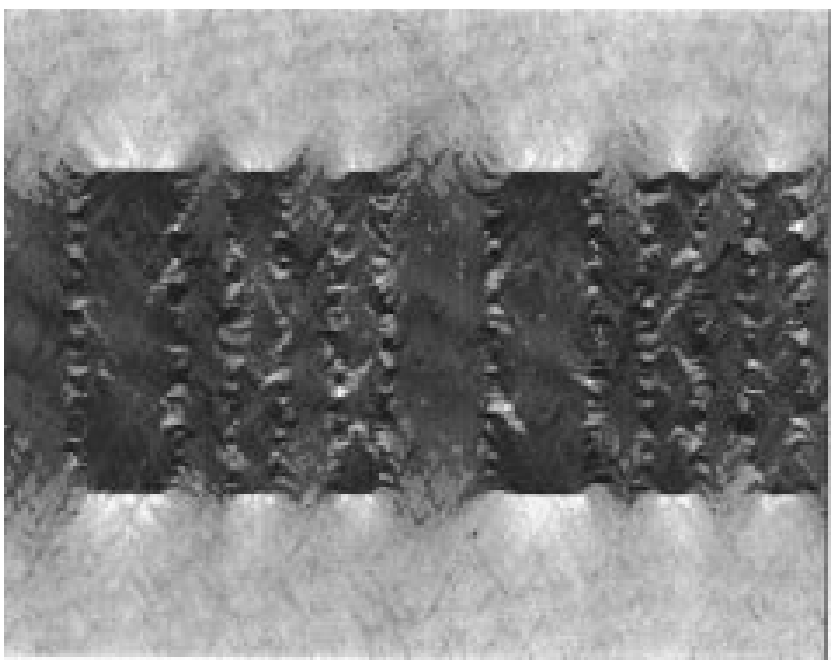

(c)

Fig. 1. Micromagnetic simulation of a recorded transition. (a) Total magnetization component along the track supporting a sequence of transitions. (b) Curl free components of magnetization. (c) Divergence free components of magnetization. transition active during the recording process. It contributes the stray field which adds to the head field during the recording process and also contributes the replayed signal.

\section{DISCUSSIONS AND OBSERVATIONS}

To illustrate how the magnetization components of Fig. 1(b) and (c) come together to produce a sawtooth-like pattern, consider the example shown in Fig. 2. Fig. 2(a) shows an arctangent magnetization distribution defined by

$$
M_{x 1}=\frac{2}{\pi} M_{r} \tan ^{-1} \frac{x}{a}
$$

where $M_{x 1}$ is the magnetization component along the track $x$ direction, $M_{r}$ is the remanent magnetization, and $a$ is the transition-width parameter which, in this case, is given a value of $0.3 \mu \mathrm{m}$. This is a curl-free distribution very reminiscent of that given in Fig. 1(b). Fig. 2(b) shows a divergence free distribution given by the simple formulas

$$
\begin{aligned}
M_{x 2} & =M_{r} \frac{b^{2}}{x^{2}+b^{2}} \cdot \cos \left(\frac{2 \pi z}{\lambda}\right) \\
M_{z} & =M_{r} \frac{b^{2} \cdot 2 x}{\left(x^{2}+b^{2}\right)^{2}} \sin \left(\frac{2 \pi z}{\lambda}\right) \cdot \frac{1}{(2 \pi / \lambda)}
\end{aligned}
$$

where $M_{x 2}$ and $M_{z}$ are its $x$ and $z$ (transverse) components of magnetization, $b$ is the width of this distribution in the track direction, and $\lambda$ is the wavelength in the cross-track dimension. The divergence-free nature of this distribution can be verified by showing that

$$
\frac{d M_{x 2}}{d x}+\frac{d M_{z}}{d z}=0
$$

Fig. 2(b), obtained for $b=3 a$ and $\lambda=1.3 \mu \mathrm{m}$, while showing a flux closure pattern also exhibits large $x$ components of magnetization. Fig. 2(c) shows the vector sum of the distributions in Fig. 2(a) and (b) using

$$
M=\left[\left(M_{x 1}+M_{x 2}\right)^{2}+M_{z}^{2}\right]^{1 / 2} .
$$

It is an excellent demonstration of how a simple transition, added to a flux-closure pattern, leads to what appears to be a sawtooth pattern. Visually there appears to be little transverse magnetization or flux closure but an impression of purely longitudinal magnetizations meeting at a sawtooth. In this transition, the arctangent (curl-free) component of the magnetization distribution produces stray fields and induces replay voltages, whereas the flux closure patterns do not.

Although it is stated above that the arctangent component of magnetization is the active part of the transition through its production of stray fields, the flux-closure patterns do remain part of the transition. Both components coexist and the vector sum of the two will add up to a magnetization equal to the saturation magnetization in the thin film medium (that is not the case in this illustration). A change in one component will lead to a change in the other and when, for example, the curlfree component of a recorded magnetization transition widens after moving away from the record head field distribution, there must be a change to the divergence-free components 


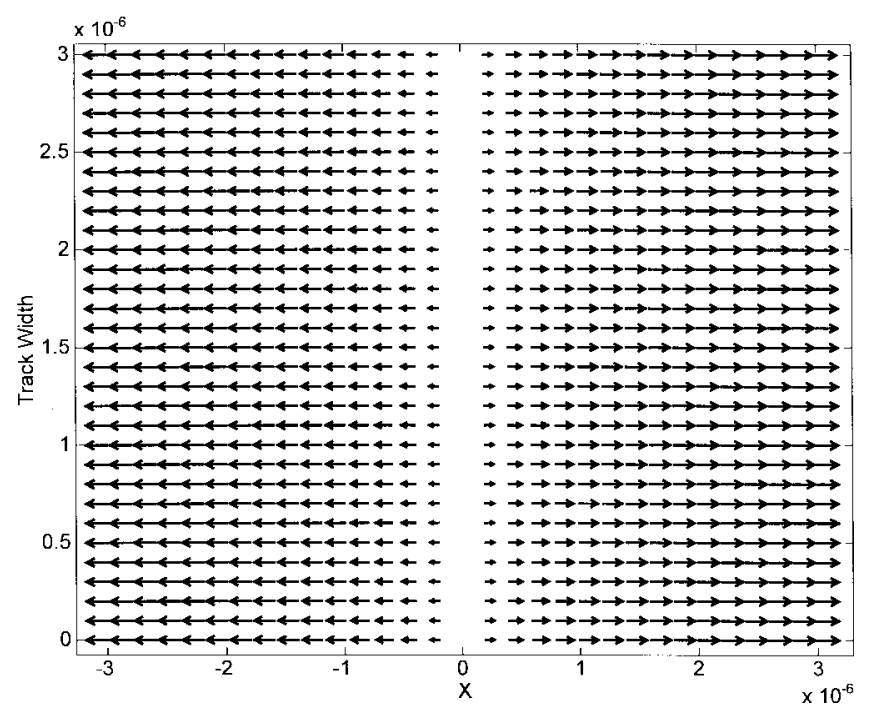

(a)

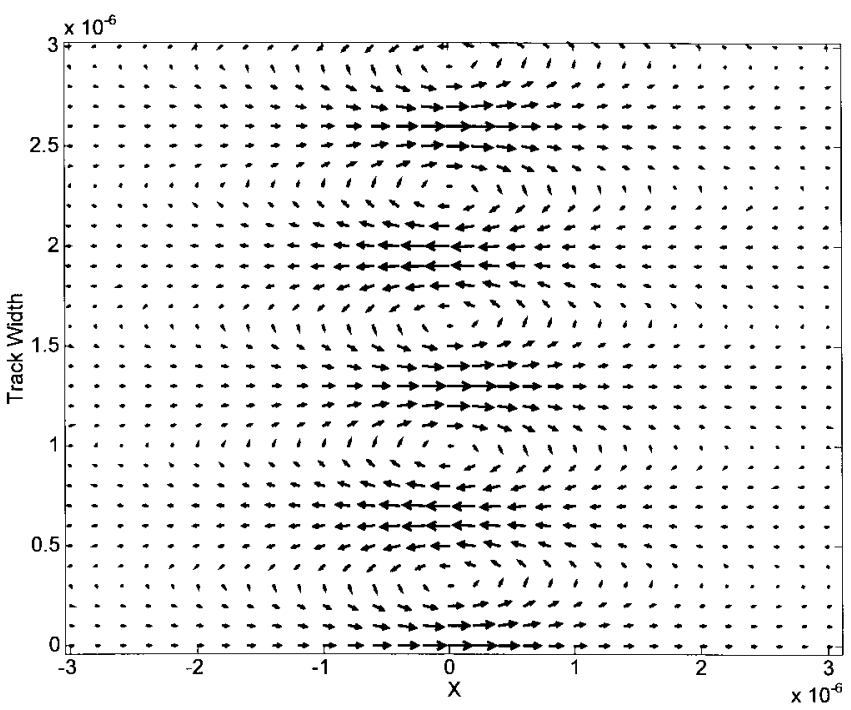

(b)

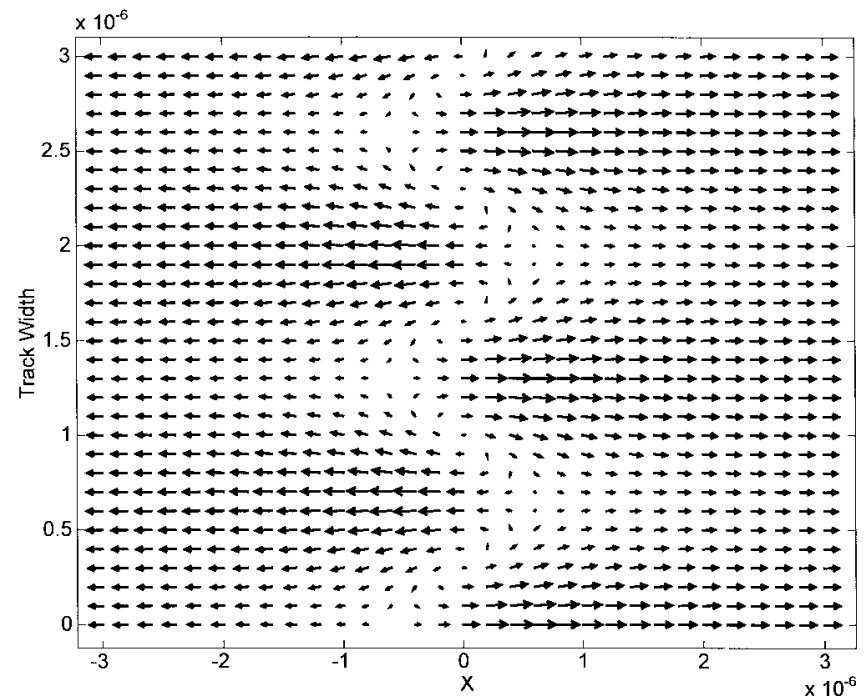

(c)

Fig. 2. (a) A magnetization distribution at an arc tangent shaped transition with $a=0.3 \mu \mathrm{m}$ (1). (b) A divergence free magnetization distribution given by (2a) and (2b) with $b=3 a$ and $\lambda=1.3 \mu \mathrm{m}$. (c) The total magnetization at a transition formed by adding the distributions of parts $2(\mathrm{a})$ and (b).

of magnetization. This is the way in which divergence-free structures originate or change.

\section{CONCLUSION}

The paper has shown that magnetization components within a transition may be thought of as a simple transition in the longitudinal component of magnetization upon which is superimposed a flux-closure pattern. Together they give the impression of sawtooth domain structures. However only the "longitudinal magnetization transition" produces a field which influences the record and replay processes and goes some way toward explaining why record and noise theory based on the arctangent magnetization have been so effective when apparent sawtooth structures are present.

These findings apply to a particular medium and micromagnetic scale where transitions are submicron in extent.

\section{REFERENCES}

[1] D. D. Dressler and J. H. Judy, "A study of digitally recorded transitions in thin magnetic films," IEEE Trans. Magn., vol. MAG-10, pp. 674-676, Sept. 1974.

[2] T. C. Arnoldussen and H. C. Tong, "Zig-zag transition profiles, noise and correlation statistics in highly oriented longitudinal thin film media," IEEE Trans. Magn., vol. MAG-22, pp. 889-891, Sept. 1986.

[3] K. Yoshiden, T. Okuwaki, N. Osakake, H. Tanabe, X. H. Onuchi, T. Matsuda, K. Shinagawa, A. Tonomura, and H. Fujiwara, "Observation of recorded magnetization patterns by electron holography," IEEE Trans. Magn., vol. MAG-19, pp. 1600-1604, Sept. 1983.

[4] B. K. Middleton, "Recording and reproducing processes," in Magnetic Recording Handbook, 2nd ed., ch. 2, C. D. Mee and E. D. Daniel, Eds. New York: McGraw-Hill, 1996.

[5] Tu Chen, "The micromagnetic properties of high coercivity metallic thin films and their effects on the limit of packing density in digital recording," IEEE Trans. Magn., vol. MAG-17, pp. 1181-1191, Mar. 1981.

[6] J. G. Zhu, "Micromagnetics of thin film media," in Magnetic Recording Technology, 2nd ed., C. D. Mee and E. D. Daniel, Eds. New York: McGraw-Hill, 1996. 
[7] J. J. Miles and B. K. Middleton, "Magnetic transition structure in longitudinal thin film media," IEEE Trans. Magn., vol. 26, pp. 204-206, Jan. 1990

[8] B. K. Middleton, "Models of the longitudinal digital magnetic recording in thin films," IEEE Trans. Magn., vol. 27, pp. 3563-3569, July 1991.

[9] M. W. Muller and E. S. Murdock, "Williams-comstock type model for sawtooth transitions in thin film media," IEEE Trans. Magn., vol. MAG-23, pp. 2368-2370, Sept. 1987.

[10] B. K. Middleton, J. J. Miles, and R. H. Noyau, "The digital recording properties of thin film media having sawtooth magnetization transitions," IEEE Trans. Magn., vol. 24, pp. 3099-3101, Nov. 1988.

[11] B. K. Middleton and J. J. Miles, "Sawtooth magnetization transitions and the digital recording properties of thin film recording media," in IEEE Conf. Proc. 319, 1991, pp. 20-25, Apr. 1990.
[12] N. R. Belk, P. K. George, and G. S. Mowry, "Noise in high performance thin film longitudinal magnetic recording media," IEEE Trans. Magn., vol. MAG-21, pp. 1350-1355, Sept. 1985.

[13] J. J. Moon, L. R. Carley, and R. R. Katti, "Density dependence of noise in thin metallic longitudinal media," J. Appl. Phys., vol. 63, pp. 3254-3256, April 1988.

[14] T. Fung, B. K. Middleton, J. J. Miles, and M. Aziz, "Transition shape and nonlinear effects in digital magnetic recording on thin film media," J. Phys. D., vol. 30, pp. 1483-1487, May 1997.

[15] M. Wdowin, J. J. Miles, B. K. Middleton, and A. Aziz, "Simulated magnetization reconstruction from DPC Lorentz and MFM measurements,' this issue, pp. 2324-2333.

[16] I. A. Beardsley, "Reconstruction of the magnetization in a thin film by a combination of Lorentz microscopy and external field measurements," IEEE Trans. Magn., vol. 25, pp. 671-677, Jan. 1989. 